\title{
Research of the deformation of a thin body with a magnetizable elastomer in the magnetic field of a coil
}

\author{
Dmitrii Merkulov ${ }^{1, *}$ \\ ${ }^{1}$ Lomonosov Moscow State University, 119991, Russia, Moscow, 1, Leninskiye Gory
}

\begin{abstract}
Deformation of a thin cylindrical body with a magnetizable elastomer based on silicone in the magnetic field of an electromagnetic coil is investigated experimentally and theoretically. It is observed that a bistability of the thin body equilibrium shape exists at some values of the coil current. The method of measuring elasticity coefficients of the magnetizable elastomer in the Mooney - Rivlin model is proposed.
\end{abstract}

\section{Introduction}

A magnetizable elastomer (ME) is a composite material consisting of elastic basis and of ferromagnetic nano- or microparticles. The ME small deformation in the applied uniform magnetic field is studied in [1-4], where only one stable equilibrium shape is found theoretically. In [5] the deformation of spherical bodies with ME in a uniform magnetic field is studied experimentally, and a unique dependence of the body length on the applied magnetic field intensity is obtained. In [6-7] the bistability of the ferrogel equilibrium shapes in a nonuniform magnetic field of an electromagnet is found experimentally. In [8-9] the possibility of bistable and of multistable equilibrium shapes of bodies with $\mathrm{ME}$ in different magnetic fields is shown theoretically.

In the present work, the possibility of bistable equilibrium shapes of the thin body with ME based on silicone (unlike the ferrogel in [6-7]) in the magnetic field of an electromagnetic coil is found experimentally. The method of measuring elasticity coefficients in the Mooney-Rivlin model is proposed. Bistability of the deformation is shown also by numerical computations.

\section{Experiment}

In the experiment, the axisymmetric cylindrical thin body with $\mathrm{ME}$ is investigated in the applied non-uniform magnetic field $\mathbf{H}_{\mathbf{0}}$, which is created by an electromagnetic coil (see Fig. 1). The magnetic field $H(x)$ on the coil axis is measured by a Hall effect sensor. The body top end is fixed at the distance of $104 \mathrm{~mm}$ from the bottom face of the coil. The point $x=0$ coincides with the point of the sample fixation. The $x$ axis is directed vertically downward. The experimental setup scheme is shown in Fig. 1.
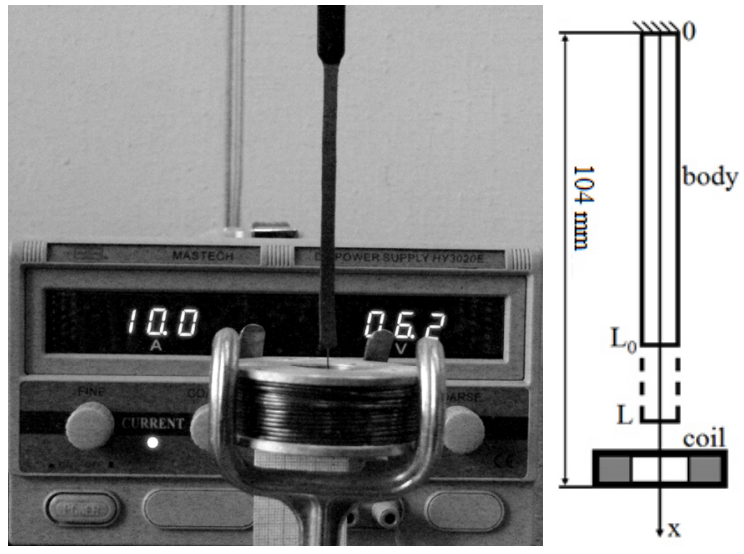

Fig. 1. Experimental setup.

In the experiment, the coil current $I$ is varied and the measurement of the body length $L$ is carried out ( $L_{0}=71 \mathrm{~mm}$ is the undeformed length of the body, the density of ME $\rho=1960 \mathrm{~kg} / \mathrm{m}^{3}$ ). The experimentally measured dependence of the relative body lengthening $\left(L-L_{0}\right) / L_{0} * 100 \%$ on the current $I$ is shown in Fig. 2.

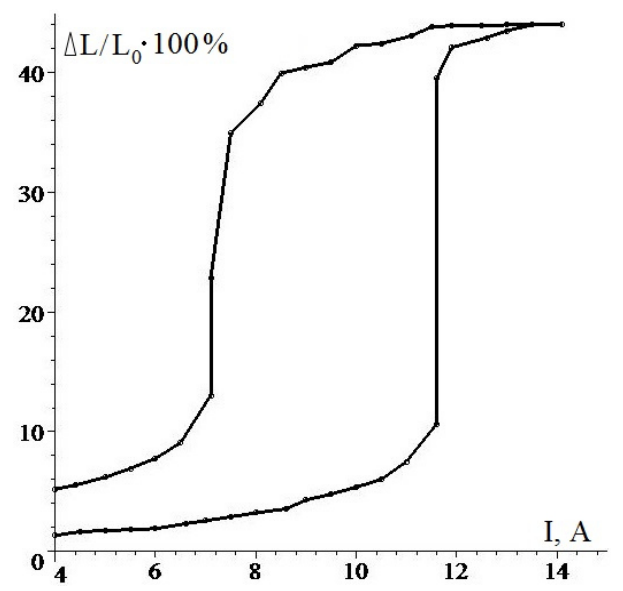

Fig. 2. The experimental dependence of the relative body lengthening on the current. 
It is seen that the shape hysteresis of the body with ME under cyclic increase and decrease of the current in the coil exists and that two stable equilibrium positions exist in some range of currents (bistability).

Measurement of the body elongation in a horizontal position upon application of forces to its ends allows to find the dependence $p_{11 n} /\left(\lambda-\lambda^{-2}\right)$ on $\lambda^{-1}\left(p_{11 n}\right.$ is a component of the stress tensor acting on the undeformed cross-section $S_{0}$ of the body; here $\lambda=L / L_{0}$, because the horizontal deformation is uniform), see Fig. 3.

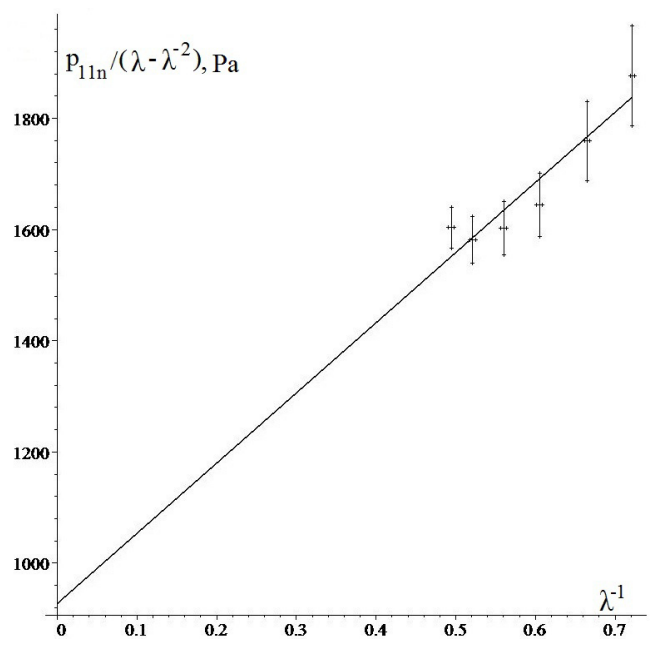

Fig. 3. The experimental dependence of $p_{11 n} /\left(\lambda-\lambda^{-2}\right)$ on $\lambda^{-1}$ for $\mathbf{H}=\mathbf{0}$.

If the elastic properties of incompressible ME are described by the Mooney - Rivlin model, the equality $p_{11 n}=2\left(\lambda-\lambda^{-2}\right)\left(C_{1}+\lambda^{-1} C_{2}\right)$ is true [10]. The graph in Fig. 3 allows to find the parameters $C_{l}=463.8 \mathrm{~Pa}$ and $C_{2}=631 \mathrm{~Pa}$ using the linear least squares approximation. In [6-7] the Mooney-Rivlin model coefficients are determined experimentally using the formula (9) in [7]. However, when a sample is compressed or stretched along the gravitational force (the samples are deformed in such way in [7]), the deformation cannot be considered homogeneous, and one cannot use the formula (9) in [7]. Perhaps the fact that for some samples the coefficient $C_{2}<0$ in [6-7] is associated with the heterogeneity of the deformations. In the present work, unlike [7], the samples based on silicone are stretched horizontally in this experiment, so the deformation is homogeneous, and one can use the formula (9) in [7]. Thus, the Mooney-Rivlin model coefficients are determined, moreover, all the coefficients are positive.

The measurement of the magnetic force, acting on a spherical body with ME in the non-uniform magnetic field of the coil, allows to calculate the magnetic permeability of the material $\mu=4.6$.

\section{Theory}

In [8] the mathematical model to calculate the length of a thin body with ME in the applied axisymmetric nonuniform magnetic field $\mathbf{H}_{\mathbf{0}}$ is proposed for the noninductive approximation. In the case of $\mathbf{M}=\chi \mathbf{H}$, $\chi=(\mu-1) /(4 \pi)=$ const, the magnetic force $\mathbf{F}_{\mathbf{m}}=M \operatorname{grad}(H)$ can be written as follows $\mathbf{F}_{\mathbf{m}}=\operatorname{grad}(G(H)), G(H)=\chi H^{2} / 2$. In the non-inductive approximation, the surface magnetic forces are small, and the magnetic force $\mathbf{F}_{\mathbf{m}}=\operatorname{grad}\left(G\left(H_{0}\right)\right)$ is a known function of coordinates. For thin enough samples, we can assume that the magnetic force inside the body is defined as $\mathbf{F}_{\mathbf{m}}=\mathbf{F}_{\mathbf{m}}(x, y=0, z=0)$. The $x$-axis is directed down along the axis of symmetry of the body. The point $x=0$ coincides with the point of the body fixation. All body surfaces are free except the surface $x=0$, so we can assume that the stress tensor components $p_{22}$ and $p_{33}$ are equal to zero on these surfaces, and the following boundary condition is true: $p_{11}(x=L)=0$. The following equations are true inside the thin body:

$$
\begin{gathered}
\frac{\partial p_{11}}{\partial x}+\frac{\partial G}{\partial x}+\rho g=0, \quad p_{22}=p_{33}=0 \\
p_{11}=2\left(\lambda^{2}-\lambda^{-1}\right)\left(C_{1}+C_{2} \lambda^{-1}\right)
\end{gathered}
$$

Here, $\lambda=\sqrt{g_{11}}=d x / d x_{0}>0, g_{11}$ is the component of the metric tensor, $x_{0}$ is the Lagrangian coordinate.

Using the equations (1), (2) and the condition $p_{I I}(L)=0$, the following equation is obtained:

$$
G(L)-G(x)+\rho g(L-x)=2\left(\lambda^{2}-\lambda^{-1}\right)\left(C_{1}+C_{2} \lambda^{-1}\right) .
$$

The first integral (3) of the static equation (2) allows to calculate $\lambda(x, L)$.

The undeformed length of the body $L_{0}$ and its length $L$ after deformation are related as follows:

$$
L_{0}=\int_{0}^{L} \frac{d x}{\lambda} .
$$

The function $\lambda(x, L)$ is determined by the equation (3), and then we can find the length of the deformed body $L$ using the equation (4).

It should be noted that in [7] the equation (20), which does not take into account the non-homogeneity of deformation, is used for the bistability theoretical research. The calculations in [7], made using the equation (20), cannot be true, because in any nonuniform magnetic field and in the gravitational field one cannot neglect the non-homogeneity of deformation. In [7] the equation (24), which describes non-homogeneous deformations in the Neo-Hooke model with the constant elasticity coefficient, is written out. In the present work, the equation (3) for statics of the elastic body in the more general Mooney - Rivlin model [10] with the constants $C_{1}$ and $C_{2}$ (which can be non-uniform in general) is obtained. In (3) in addition to the magnetic force, gravity force is also taken into account.

\section{The numerical calculations}

The potential $G(x)$ can be calculated by using the experimentally measured distribution of the magnetic field of the coil $H(x)$. The calculations using the 
experimentally measured parameters $(\mu=4.6$, $\left.\rho=1960 \mathrm{~kg} / \mathrm{m}^{3}, C_{1}=463.8 \mathrm{~Pa}, C_{2}=631 \mathrm{~Pa}\right)$ show that for some values of the current in the electromagnetic coil, the dependence of $L$ on $L_{0}$ is ambiguous. Three values of $L$ correspond to one value $L_{0}$ (in some range of the current $I$, see Fig. 4).

It is possible to show that if $d L / d L_{0}<0$ the equilibrium state is unstable [9]. So, the middle of these three values $L$ corresponds to the unstable equilibrium state because the $d L / d L_{0}<0$ at this branch of the solution. Thus, the existence of the bistable body deformation in some current range is shown theoretically. Some quantitative difference between theory and experiment may be due to the fact that in calculations the dependence of $C_{1}$ and $C_{2}$ on the magnitude of the magnetic field is not taken into account.

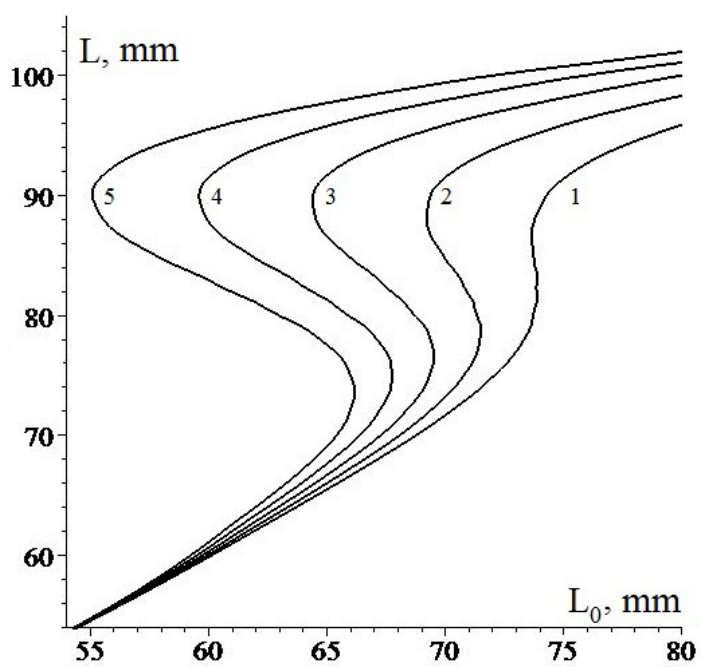

Fig. 4. The theoretical dependence of the deformed body length on its initial length at currents: $1-6 \mathrm{~A}, 2-7 \mathrm{~A}, 3-8 \mathrm{~A}$, $4-9$ A, 5-10 A.

\section{Conclusion}

The deformation of a thin cylindrical body with the magnetizable elastomer based on silicone in the applied non-uniform axisymmetric magnetic field of the electromagnetic coil is investigated experimentally and theoretically. The bistable deformation of such body in some range of currents in the coil is observed in the experiment. The magnetic permeability of the magnetizable elastomer is measured by the power method. The technique of measuring elasticity coefficients of the elastomer in the Mooney-Rivlin model is proposed. The numerical calculations with the experimental data show that in some current range the bistability of the body equilibrium shapes exists. Some quantitative difference between theory and experiment may be due to various reasons, for example, because the dependence of $C_{1}$ and $C_{2}$ on the magnitude of the magnetic field is not taken into account in calculations.

This work is supported by the Russian Foundation for Basic Research (16-51-12024), the Russian President grant MK4541.2016.1 and the FASIE (8541ГУ/2015).

\section{References}

1. O.V. Stolbov, Yu.L. Raikher, Tech. Phys. Lett., 26(2), 156-158 (2000)

2. G.V. Stepanov, Yu.L. Raikher, O.V. Stolbov, Tech. Phys., 53(9), 1169-1176 (2008)

3. K. Morozov, M. Shliomis, H. Yamaguchi, Phys. Rev. E, 79, 040801: 1-4 (2009)

4. D. Ivaneyko, V. Toshchevikov, M. Saphiannikova, G. Heinrich, Cond. Matt. Phys., 15(3), 33601: 1-12 (2012)

5. L.V. Nikitin, K.G. Kornev, L.S. Mironova, VIII Plyos international conference on magnetic fluids (1998, in Russian), 95-96

6. M. Zrinyi, L. Barsi, D. Szabo, H.-G. Kilian, J. Chem. Phys., 106, 5685-5692 (1997)

7. G. Filipcsei, I. Csetneki, A. Szilágyi, M. Zrínyi, Adv. Polym. Sci., 206, 137-189 (2007)

8. V.A. Naletova, D.A. Pelevina, D.I. Merkulov, I. Zeidis, K. Zimmermann, Magnetohydrodynamics, 52(3), 287-298 (2016)

9. D.I. Merkulov, V.A. Naletova, D.A. Pelevina, V.A. Turkov, JMMM, 431, 123-125 (2017)

10. G.V. Vinogradov, A.Y. Malkin. Rheology of Polymers. Springer-Verlag Berlin Heidelberg, 1980, p. 468 